\title{
Summary for policymakers of the thematic assessment on pollinators, pollination and food production
}

\section{Key messages}

\section{Values of pollinators and pollination}

1. Animal pollination plays a vital role as a regulating ecosystem service in nature. Globally, nearly 90 per cent of wild flowering plant species depend, at least in part, on the transfer of pollen by animals. These plants are critical for the continued functioning of ecosystems as they provide food, form habitats, and provide other resources for a wide range of other species.

2. More than three quarters of the leading types of global food crops rely to some extent on animal pollination for yield and/or quality. Pollinator-dependent crops contribute to 35 per cent of global crop production volume.

3. Given that pollinator-dependent crops rely on animal pollination to varying degrees, it is estimated that 5-8 per cent of current global crop production is directly attributed to animal pollination with an annual market value of \$235 billion-\$577 billion (in 2015, United States dollars ${ }^{1}$ ) worldwide.

4. The importance of animal pollination varies substantially among crops, and therefore among regional crop economies. Many of the world's most important cash crops benefit from animal pollination in terms of yield and/or quality and are leading export products in developing countries (e.g., coffee and cocoa) and developed countries (e.g., almond), providing employment and income for millions of people.

5. Pollinator-dependent food products are important contributors to healthy human diets and nutrition. Pollinatordependent species encompass many fruit, vegetable, seed, nut and oil crops, which supply major proportions of micronutrients, vitamins, and minerals in the human diet.

6. The vast majority of pollinator species are wild, including more than 20,000 species of bees, and some species of flies, butterflies, moths, wasps, beetles, thrips, birds and bats and other vertebrates. A few species of bees are widely managed, including the western honey bee $^{2}$. (Apis mellifera), the eastern honey bee (Apis cerana), some bumble bees, some stingless bees, and a few solitary bees. Beekeeping provides an important source of income for many rural livelihoods. The western honey bee is the most widespread managed pollinator in the world, and globally there are about 81 million hives producing an estimated 1.6 million tonnes of honey annually.

7. Both wild and managed pollinators have a globally significant role in crop pollination, although their relative contributions differ according to crop and location. Crop yield and/or quality depends on both the abundance and diversity of pollinators. A diverse community of pollinators generally provides more effective and stable crop pollination than any

\footnotetext{
${ }^{1}$ Value adjusted to 2015 United States dollars taking into account inflation only.

${ }^{2}$ Also called the European honey bee, native to Africa, Europe and Western Asia, but spread around the globe by beekeepers and queen breeders.
}

single species. Pollinator diversity contributes to crop pollination even when managed species (e.g., honey bees) are present in high abundance. The contribution of wild pollinators to crop production is undervalued.

8. Pollinators are a source of multiple benefits to people, beyond food provisioning, contributing directly to medicines, biofuels (e.g. canola ${ }^{3}$, palm oil), fibres (e.g, cotton, linen) construction materials (timbers), musical instruments, arts and crafts, recreational activities and as sources of inspiration for art, music, literature, religion, traditions, technology and education. Pollinators serve as important spiritual symbols in many cultures. Sacred passages about bees in all the worlds' major religions highlight their significance to human societies over millennia.

9. A good quality of life for many people relies on ongoing roles of pollinators in globally significant heritage; as symbols of identity; as aesthetically significant landscapes and animals; in social relations; for education and recreation; and governance interactions. Pollinators and pollination are critical to the implementation of: the Convention for the Safeguarding of the Intangible Cultural Heritage (UNESCO); the Convention Concerning the Protection of the World Cultural and Natural Heritage (UNESCO); and Globally Important Agricultural Heritage Systems (FAO).

\section{Status and trends in pollinators and pollination}

10. Wild pollinators have declined in occurrence and diversity (and abundance for certain species) at local and regional scales, in North West Europe and North America. Although a lack of wild pollinator data (species identity, distribution and abundance) for Latin America, Africa, Asia and Oceania preclude any general statement on their regional status, local declines have been recorded. Long-term international or national monitoring of both pollinators and pollination is urgently required to provide information on status and trends for most species and most parts of the world.

11. The number of managed western honey bee hives has increased globally over the last five decades, even though declines have been recorded in some European countries and North America over the same period. Seasonal colony loss of western honey bees has in recent years been high at least in some parts of the temperate Northern Hemisphere and in South Africa. Beekeepers can under some conditions, with associated economic costs, make up such losses through splitting of managed colonies.

12. The International Union for Conservation of Nature (IUCN) Red List assessments indicate that $\mathbf{1 6 . 5}$ per cent of vertebrate pollinators are threatened with global extinction (increasing to 30 per cent for island species). There are no global Red List assessments specifically for insect pollinators. However, regional and national assessments indicate high levels of threat for some bees and butterflies. In Europe, 9 per cent of bee and butterfly species are threatened and populations are declining

\footnotetext{
${ }^{3}$ Also called oil seed rape
} 
for 37 per cent of bees and 31 per cent of butterflies (excluding data deficient species, which includes 57 per cent of bees). Where national Red List assessments are available, they show that often more than 40 per cent of bee species may be threatened.

13. The volume of production of pollinator-dependent crops has increased by $\mathbf{3 0 0}$ per cent over the last five decades making livelihoods increasingly dependent on the provision of pollination. However, overall these crops have experienced lower growth and lower stability of yield than pollinator-independent crops. Yield per hectare of pollinator-dependent crops has increased less, and varies more year to year than yield per hectare of pollinator-independent crops. While the drivers of this trend are not clear, studies of several crops at local scales show that production declines when pollinators decline.

\section{Drivers of change, risks and opportunities, and policy and management options}

14. The abundance, diversity and health of pollinators and the provision of pollination are threatened by direct drivers which generate risks to societies and ecosystems. Threats include landuse change, intensive agricultural management and pesticide use, environmental pollution, invasive alien species, pathogens and climate change. Explicitly linking pollinator declines to individual or combinations of direct drivers is limited by data availability or complexity, yet a wealth of individual case studies worldwide suggests that these direct drivers often affect pollinators negatively.

15. Strategic responses to the risks and opportunities associated with pollinators and pollination range in ambition and timescale, from immediate, relatively straightforward responses that reduce or avoid risks, to larger scale and longer-term responses that aim to transform agriculture, or society's relationship with nature. There are seven broad strategies, linked to actions, for responding to risks and opportunities, including a range of solutions that draw on indigenous and local knowledge. These strategies can be adopted in parallel, and would be expected to reduce risks associated with pollinator decline in any region of the world, regardless of the extent of available knowledge about the status of pollinators or the effectiveness of interventions.

16. A number of features of current intensive agricultural practices threaten pollinators and pollination. Moving towards more sustainable agriculture and reversing the simplification of agricultural landscapes offer key strategic responses to risks associated with pollinator decline. Three complementary approaches to maintaining healthy pollinator communities and productive agriculture are: (a) ecological intensification (i.e., managing nature's ecological functions to improve agricultural production and livelihoods while minimizing environmental damage); (b) strengthening existing diversified farming systems (including forest gardens, home gardens, agroforestry and mixed cropping and livestock systems) to foster pollinators and pollination through practices validated by science or indigenous and local knowledge (e.g., crop rotation); and (c) investing in ecological infrastructure by protecting, restoring and connecting patches of natural and semi-natural habitats throughout productive agricultural landscapes. These strategies can concurrently mitigate the impacts of land-use change, land management intensity, pesticide use and climate change on pollinators.
17. Practices based on indigenous and local knowledge, in supporting an abundance and diversity of pollinators can, in coproduction with science, be a source of solutions to current challenges. Practices include diverse farming systems; favouring heterogeneity in landscapes and gardens; kinship relationships that protect many specific pollinators; using seasonal indicators (e.g., flowering) to trigger actions (e.g., planting); distinguishing a wide range of pollinators; and tending to nest trees, and floral and other pollinator resources. Knowledge co-production has led to improvements in hive design; new understanding of parasite impacts; and the identification of stingless bees new to science.

18. The risk to pollinators from pesticides is through a combination of the toxicity and the level of exposure, which varies geographically with compounds used, and the scale of land management and habitat in the landscape. Pesticides, particularly insecticides, have been demonstrated to have a broad range of lethal and sublethal effects on pollinators in controlled experimental conditions. The few available field studies assessing effects of field-realistic exposure provide conflicting evidence of effects based on species studied and pesticide usage. It is currently unresolved how sublethal effects of pesticide exposure recorded for individual insects affect colonies and populations of managed bees and wild pollinators, especially over the longer-term. Recent research focusing on neonicotinoid insecticides shows evidence of lethal and sublethal effects on bees and some evidence of impacts on the pollination they provide. There is evidence from a recent study which shows impacts of neonicotinoids on wild pollinator survival and reproduction at actual field exposure. ${ }^{4}$ Evidence, from this and other studies, for effects on managed honey bee colonies is conflicting

19. Exposure of pollinators to pesticides can be decreased by reducing the use of pesticides seeking alternative forms of pest control, and adopting a range of specific application practices, including technologies to reduce pesticide drift. Actions to reduce pesticide use include promoting Integrated Pest Management supported by educating farmers, organic farming and policies to reduce overall use. Risk assessment can be an effective tool to define pollinator-safe uses of pesticides, which should consider different levels of risk among wild and managed pollinator species according to their biology. Subsequent use regulations (including labelling) are important steps towards avoiding the misuse of specific pesticides. The International Code of Conduct on the Distribution and Use of Pesticides of the Food and Agriculture Organization of the United Nations (FAO) provides a set of voluntary actions for Government and industry to reduce risks for human health and environment, although only 15 per cent of countries are using this. ${ }^{5}$

20. Most agricultural genetically modified organisms (GMOs) carry traits for herbicide tolerance (HT) or insect resistance (IR). Reduced weed populations are likely to accompany most HT crops, diminishing food resources for pollinators. The actual consequences for the abundance and diversity of pollinators foraging in HT-crop fields is unknown. IR crops can result in the reduction of insecticide use which varies regionally according

\footnotetext{
${ }^{4}$ Rundlof et al., 2015. Seed coating with a neonicotinoid insecticide negatively affects wild bees. Nature 521: 77-80 doi: 10.1038/nature14420.

${ }^{5}$ Based on a survey from 2004-2005; Ekström, G., and Ekbom, B. 2010. Can the IOMC Revive the 'FAO Code' and take stakeholder initiatives to the developing world? Outlooks on Pest Management 21:125-131.
} 
to the prevalence of pests, the emergence of secondary outbreaks of non-target pests or primary pest resistance. If sustained, this reduction in insecticide use could reduce this pressure on nontarget insects. How IR-crop use and reduced pesticide use affect pollinator abundance and diversity is unknown. Risk assessment required for the approval of GMO crops in most countries does not adequately address the direct sublethal effects of IR crops or the indirect effects of HT and IR crops, partly because of the lack of data.

21. Bees suffer from a broad range of parasites, including Varroa mites in western and eastern honey bees. Emerging and re-emerging diseases are a significant threat to the health of honey bees, bumble bees and solitary bees especially when managed commercially. Greater emphasis on hygiene and the control of pathogens would help reduce the spread of disease across the entire community of pollinators, managed and wild. Mass breeding and large-scale transport of managed pollinators can pose risks for the transmission of pathogens and parasites, and increase the likelihood of selection for more virulent pathogens, alien species invasions, and regional extinctions of native pollinator species. The risk of unintended harm to wild and managed pollinators could be decreased by better regulation of their trade and use.

22. The ranges, abundances, and seasonal activities of some wild pollinator species (e.g., bumble bees and butterflies) have changed in response to observed climate change over recent decades. Generally, the impacts of ongoing climate change on pollinators and pollination services to agriculture may not be fully apparent for several decades, owing to a delayed response in ecological systems. Adaptive responses to climate change include increasing crop diversity and regional farm diversity, and targeted habitat conservation, management or restoration. The effectiveness of adaptation efforts at securing pollination under climate change is untested. Many actions to support wild and managed pollinators and pollination could be implemented more effectively with improved governance. For example, broad-scale government policy may be too homogenous and not allow for local variation in practices; administration can be fragmented into different levels; and goals can be contradictory between sectors. Coordinated, collaborative action and knowledge-sharing that builds links across sectors (e.g., agriculture and nature conservation), across jurisdictions (e.g., private, government, not-for-profit), and among levels (e.g., local, national, global) can overcome these challenges and lead to long-term changes that benefit pollinators. Establishing effective governance requires habits, motivations and social norms to change over the long term. However, the possibility that contradictions between policy sectors remain even after coordination efforts should be acknowledged and be a point of attention in future studies.

Drafting authors: Simon G. Potts, Vera Imperatriz-Fonseca, Hien T. Ngo, Jacobus C. Biesmeijer, Thomas D. Breeze, Lynn V. Dicks, Lucas A. Garibaldi, Rosemary Hill, Josef Settele and Adam J. Vanbergen.

\section{Intergovernmental Science-Policy Platform on Biodiversity and Ecosystem Services - IPBES}

